\title{
Evaluation of Matrix Metalloproteinase 9 Serum Concentration as a Biomarker in Malignant Mesothelioma
}

\author{
Danijela Štrbac $\mathbb{D}^{1},{ }^{1}$ Katja Goričar $\mathbb{D}^{2},{ }^{2}$ Vita Dolžan $\mathbb{D},{ }^{2}$ and Viljem Kovač $\mathbb{D}^{1}$ \\ ${ }^{1}$ Institute of Oncology Ljubljana, Zaloška 2, 1000 Ljubljana, Slovenia \\ ${ }^{2}$ University of Ljubljana, Faculty of Medicine, Institute of Biochemistry, Pharmacogenetics Laboratory, Vrazov trg 2, \\ 1000 Ljubljana, Slovenia
}

Correspondence should be addressed to Viljem Kovač; vkovac@onko-i.si

Received 10 January 2019; Revised 14 March 2019; Accepted 3 April 2019; Published 2 May 2019

Academic Editor: Rudy Foddis

Copyright ( 2019 Danijela Štrbac et al. This is an open access article distributed under the Creative Commons Attribution License, which permits unrestricted use, distribution, and reproduction in any medium, provided the original work is properly cited.

Background. Malignant mesothelioma (MM) is a rare, but fatal disease with few treatment options. The diagnosis and treatment response are challenging in MM. Therefore, the search for novel diagnostic and prognostic biomarkers is ongoing. The aim of our study was to investigate matrix metalloproteinase 9 (MMP9) as a potential serum biomarker of treatment response and survival in MM. We also investigated the influence of genetic polymorphisms on MMP9 serum levels. Methods. We included 110 patients with MM that have been previously genotyped for common MMP9 polymorphisms. Serum samples were collected before treatment, at the end of chemotherapy, and at the time of progression. MMP9 serum levels were measured using enzyme-linked immunosorbent assay kits. The role of serum MMP9 and MMP9 polymorphisms in treatment response was determined using the nonparametric tests and logistic or Cox regression. Results. Median serum MMP9 was 706.7 (499.6-1224.9) $\mathrm{ng} / \mathrm{ml}$ before treatment, 440.5 (255.9-685.2) $\mathrm{ng} / \mathrm{ml}$ after chemotherapy, and $502.8(307.2-851.4) \mathrm{ng} / \mathrm{ml}$ at disease progression. After chemotherapy, $87(79.8 \%)$ patients had lower serum MMP9, with the median change of -286.3 $(-607.3$ to -70.2$) \mathrm{ng} / \mathrm{ml}(P<0.001)$. At disease progression, $47(65.3 \%)$ patients had lower serum MMP9 compared to pretreatment values, with the median change of $-163.7(-466.6$ to 108.6$) \mathrm{ng} / \mathrm{ml}(P=0.001)$. Patients with higher performance status had higher serum MMP9 before treatment $(P=0.010)$. Among investigated polymorphisms, only rs17576 was associated with serum MMP9 levels before treatment $(P=0.041)$. Conclusion. Median serum MMP9 levels differed significantly before and after treatment of MM, but failed to reach significance as a standalone biomarker. The contribution of MMP9 serum levels and MMP9 polymorphisms to a composite diagnostic and prognostic biomarker should be further tested.

\section{Introduction}

Malignant mesothelioma (MM) is a rare but fatal disease, with limited treatment options; however, new targeted therapies are emerging [1]. In the recent years, extensive efforts have been put into finding novel treatment options, as well as novel diagnostic, prognostic, and predictive biomarkers [2]. An ongoing search for the last thirty years has identified mesothelin (soluble mesothelin-related peptides, SMRP) as a promising clinically relevant biomarker for monitoring the response and disease progression in MM [3]. SMRP in pleural fluid has been suggested to distinguish pleural mesothelioma from pleural metastasis and therefore aid in the diagnosis of MM $[4,5]$. Additional serum peptides, such as survivin and fibulin-3, have been investigated in an effort to find new biomarkers that would further support the diagnostic and treatment process in MM [6, 7]. Serum survivin decreased significantly with good response to treatment [6], while higher serum levels of fibulin-3 were found in patients that progressed within 18 months of treatment [7].

As none of these serum biomarkers demonstrated a high enough sensitivity as a standalone marker in mesothelioma, several attempts were made to improve the sensitivity and specificity [8-10]. For example, single nucleotide polymorphisms (SNPs) in the mesothelin gene (MSLN) could improve the prognostic value of mesothelin [11-13]. On the other hand, additional serum markers were studied. A study that investigated SMRP as well as matrix 
metalloproteinase 9 (MMP9) among several potential biomarkers concluded that the combination of radiographic findings and blood markers could be used to stratify the risk of MM in asbestos-exposed population [14]. Furthermore, MMP9 overexpression in correlation with MSLN overexpression showed increased tumor invasion and decreased survival in MM patients [15].

MMP9 was therefore investigated as a potential biomarker in MM, but its association with clinical outcome has not been elucidated in MM. There are substantial data of biological function of MMP9 in all thoracic malignancies. MMP9 is involved in turnover and remodeling of the extracellular matrix in normal respiratory epithelial growth, suggesting a potential role in carcinogenesis. Healthy lung tissue in itself has an intrinsic, basal expression of MMP9. The stromal cells overexpress MMP9 in inflammatory disease, albeit with a greater expression in tumor cells $[15,16]$. However, MMP9 and MMP2 as potential biomarkers in MM did not reach diagnostic value as potential standalone biomarkers and more studies are needed to evaluate the role of soluble MMP9 in MM. We have shown that MMP9 rs2250889 CG genotype carriers had significantly shorter overall survival (OS) and time to progression (TTP), while MMP9 rs20544 CT allele carriers had longer OS $[17,18]$.

These results led us to further investigate if these polymorphisms influence MMP9 serum levels. Furthermore, we have assessed the predictive values of MMP9 serum levels in MM patients before and after treatment as well as at disease progression.

\section{Patients and Methods}

2.1. Patients. Patients with histologically proven pleural or peritoneal mesothelioma diagnosed and treated between 2007 and 2016 were included in this retrospective study. The patients were diagnosed at the University Clinic Golnik and University Clinic Ljubljana, Department of Thoracic Surgery. Most of the patients were treated and followed up at the Institute of Oncology, Ljubljana. Patients received chemotherapy according to standard protocols that consist of six cycles of gemcitabine/cisplatin (carboplatin) or pemetrexed/ cisplatin (carboplatin) chemotherapy. Patients did not receive any maintenance chemotherapy.

Most patients included in the study were also participating in previous studies on pharmacogenomics of MPM treatment, at the Institute of Oncology, Ljubljana. Some of the patients were included in a parallel clinical trial AGILI (Trial registration ID: NCT01281800).

The study was approved by the Slovenian Ethics Committee for Research in Medicine and was carried out according to the Declaration of Helsinki.

2.2. Experimental Methods. Serum samples were collected before treatment, at the end of chemotherapy, and at the time of progression. We included in the study only MM patients that had available serum samples before treatment and at least one additional sample (at the end of chemotherapy or at disease progression or both). For samples at the end of chemotherapy, blood was sampled on the day of the last (6th) chemotherapy cycle, unless disease progression occurred before the last cycle. None of the patients received maintenance chemotherapy. Serum samples were prepared immediately after blood sampling, aliquoted, and stored at $-20^{\circ} \mathrm{C}$ until MMP9 levels were measured. The remaining blood peripheral lymphocytes were used for DNA isolation, and all the patients have been genotyped for common $M M P 2, M M P 9$, and MMP14 polymorphisms [17].

MMP9 serum levels were measured using enzyme-linked immunosorbent assay (ELISA) kits according to the manufacturer's instructions (Quantikine ELISA, R\&D Systems, Minneapolis, USA). This assay enables detection of natural human and recombinant MMP9, both the $92 \mathrm{kDa}$ ProMMP9 form and the active $82 \mathrm{kDa}$ MMP9 form [19]. Minimum detectable dose of the assay is $0.156 \mathrm{ng} / \mathrm{ml}$. The manufacturer of the assay reports the intra-assay coefficient of variation $(\mathrm{CV})$ for MMP-9 as $1.9-2.9 \%$ and interassay $\mathrm{CV}$ as $6.9-7.9 \%$. In our study, serum samples were diluted 100 -fold. The standard curve was calculated using a fourparameter logistic curve fit.

2.3. Statistical Analyses. Continuous and categorical variables were described using median with interquartile range (25\%-75\%) and frequencies, respectively. The nonparametric Mann-Whitney or Kruskal-Wallis tests were used to compare distribution of continuous variables between different categories for independent samples, while the Wilcoxon test was used for related samples. Pairwise comparisons with post hoc Bonferroni corrections were used with the KruskalWallis test. Spearman's rho was used to assess correlations between continuous variables. The association of serum MMP9 levels with response rate was determined using logistic regression, while survival analysis was performed using Cox regression to calculate hazard ratios (HRs) and their 95\% confidence intervals (CIs). The Kaplan-Meier analysis was used to calculate the median survival and follow-up times. The additive and dominant genetic models were used in analyses assessing the association of SNPs with serum MMP9 concentration. Deviation from the Hardy-Weinberg equilibrium (HWE) was evaluated using the chi-square test. All statistical analyses were carried out using IBM SPSS Statistics, version 21.0 (IBM Corporation, Armonk, NY, USA). Haplotypes were reconstructed and analyzed using THESIAS software where the most frequent haplotype served as the reference. All statistical tests were two sided, and the level of significance was set to 0.05 .

\section{Results}

In total, $110 \mathrm{MM}$ patients were included in the study. Their characteristics are summarized in Table 1. All patients were treated with chemotherapy; $66(60 \%)$ received gemcitabine/cisplatin (carboplatin) as first-line chemotherapy, 41 (37.3\%) received pemetrexed/cisplatin (carboplatin), and $3(2.7 \%)$ received other types of chemotherapy. 81 (73.6\%) patients had epithelioid histological type, and 56 patients had Eastern Cooperative Oncology Group (ECOG) (50.9\%) performance status of 1 . 
TABLe 1: Patients' characteristics $(N=110)$ and their association with serum MMP9 before treatment.

\begin{tabular}{|c|c|c|c|c|}
\hline Characteristic & & $N(\%)$ & $\begin{array}{l}\text { MMP9 before treatment }(\mathrm{ng} / \mathrm{ml}) \\
\text { Median }(25 \%-75 \%)\end{array}$ & $P$ \\
\hline \multirow{2}{*}{ Gender } & Male & $89(80.9)$ & $707.8(502.3-1224.7)$ & \multirow[t]{2}{*}{$0.651^{\mathrm{a}}$} \\
\hline & Female & $21(19.1)$ & $683.5(404.1-1231.8)$ & \\
\hline Age & Median (25\%-75\%) (years) & $66(59.8-72.3)$ & Spearman's rho $=-0.036$ & $0.410^{\mathrm{b}}$ \\
\hline \multirow{5}{*}{ Stage } & I & $12(10.9)$ & $595.8(502.6-1412.1)$ & \multirow[t]{5}{*}{$0.982^{\mathrm{a}}$} \\
\hline & II & $22(20.0)$ & $729.0(396.8-1216.0)$ & \\
\hline & III & $42(38.2)$ & $772.3(452.6-1277.5)$ & \\
\hline & IV & $27(24.5)$ & $698.5(548.9-1207.2)$ & \\
\hline & Peritoneal & $7(6.4)$ & $757.4(632.5-1511.6)$ & \\
\hline \multirow{4}{*}{ Histological type } & Epithelioid & $81(73.6)$ & $757.4(497.6-1213.2)$ & \multirow[t]{4}{*}{$0.830^{\mathrm{a}}$} \\
\hline & Biphasic & $15(13.6)$ & $683.5(393.3-1408.9)$ & \\
\hline & Sarcomatoid & $11(810.0)$ & $641.3(483.4-1178.1)$ & \\
\hline & Not characterized & $3(2.7)$ & $705.7(698.5-705.7)$ & \\
\hline \multirow{3}{*}{ ECOG performance status } & 0 & $13(11.8)$ & $466.7(383.2-842.5)$ & \multirow[t]{3}{*}{$0.010^{\mathrm{a}}$} \\
\hline & 1 & $56(50.9)$ & $682.0(431.5-1088.4)$ & \\
\hline & 2 & $41(37.3)$ & $1026.5(579.9-1443.0)$ & \\
\hline CRP & Median (25\%-75\%) (mg/l) & $17.5(6.3-54.3)[6]$ & Spearman's rho $=0.067$ & $0.498^{\mathrm{b}}$ \\
\hline $\mathrm{LDH}$ & Median $(25 \%-75 \%)(\mu \mathrm{kat} / \mathrm{l})$ & $2.6(2.2-2.9)[6]$ & Spearman's rho $=-0.066$ & $0.505^{\mathrm{b}}$ \\
\hline \multirow{2}{*}{ Asbestos exposure } & Not exposed & $24(21.8)$ & $644.1(502.6-1238.1)$ & \multirow[t]{2}{*}{$0.625^{\mathrm{a}}$} \\
\hline & Exposed & $86(78.2)$ & $749.1(497.1-1216.0)$ & \\
\hline \multirow{2}{*}{ Smoking } & Nonsmokers & $61(56.0)[1]$ & $707.8(406.6-1293.5)$ & \multirow[t]{2}{*}{$0.732^{\mathrm{a}}$} \\
\hline & Smokers & $48(44.0)$ & $687.7(558.0-1199.9)$ & \\
\hline \multirow{2}{*}{ Pain } & No & $50(45.9)[1]$ & $641.3(497.1-1243.9)$ & \multirow[t]{2}{*}{$0.349^{\mathrm{a}}$} \\
\hline & Yes & $59(54.1)$ & $800.2(546.1-1242.3)$ & \\
\hline \multirow{2}{*}{ Weight loss } & No & $32(29.6)[2]$ & $868.9(567.9-1175.4)$ & \multirow[t]{2}{*}{$0.699^{\mathrm{a}}$} \\
\hline & Yes & $76(70.4)$ & $682.0(485.9-1357.7)$ & \\
\hline
\end{tabular}

Numbers in [] denote missing data. ${ }^{a}$ Calculated using the Mann-Whitney or Kruskal-Wallis test. ${ }^{b}$ Calculated using Spearman's rho. CRP: C-reactive protein; ECOG: Eastern Cooperative Oncology Group; LDH: lactate dehydrogenase.

At the time of data evaluation, 95 (86.4\%) of the patients experienced disease progression and 60 (54.5\%) of the included patients were dead. Median TTP from the beginning of chemotherapy was 7.9 (5.4-14.7) months, OS was 21.8 (10.1-28.8) months, and follow-up time from the beginning of chemotherapy was 21.8 (13.8-74.9) months as well. Serum samples after chemotherapy were available for 109 out of 110 patients, while serum samples at disease progression were available for 72 out of 95 patients.

In ELISA assay, average intra-assay CV for our samples measured in duplicates on the same plate was $5.7 \%$, while interassay CV was $10 \%$. As intra-assay CV was below $10 \%$ and interassay CV was below 15\%, the variation between measurements was sufficiently low.

Median serum MMP9 before treatment was 706.7 (499.61224.9) $\mathrm{ng} / \mathrm{ml}$, after chemotherapy 440.5 (255.9-685.2) $\mathrm{ng} / \mathrm{ml}$, and at disease progression $502.8(307.2-851.4) \mathrm{ng} / \mathrm{ml}$. After chemotherapy, 87 (79.8\%) patients had lower serum MMP9 compared to pretreatment values, with the median change of $-286.3(-607.3$ to -70.2$) \mathrm{ng} / \mathrm{ml}(P<0.001)$. At disease progression, serum MMP9 level was significantly lower compared to pretreatment values $(P=0.001)$. At progression, 47 (65.3\%) patients had lower serum MMP9 compared to pretreatment values, with the median change of -163.7 (-466.6 to 108.6$) \mathrm{ng} / \mathrm{ml}$. On the other hand, serum MMP9 levels were statistically significantly higher at disease progression compared to values after chemotherapy $(P=0.006)$. At disease progression, only $17(23.6 \%)$ patients had lower MMP9, while 36 (50.0\%) patients had slightly higher serum MMP9 compared to values after chemotherapy, with the median change of $2.7(0.0-372.7) \mathrm{ng} / \mathrm{ml}$.

The impact of clinical characteristics on serum MMP9 levels is presented in Table 1. Patients with higher ECOG performance status had higher serum MMP9 before treatment $(P=0.010)$. The difference between patients with ECOG status 2 and patients with ECOG status 0 was statistically significant even after post hoc Bonferroni correction $(P=0.018)$. Serum MMP9 levels did not differ between different histological types $(P=0.830)$.

There was no significant difference in serum MMP9 levels before and after treatment in patient groups with different responses to treatment (Table 2).

Compared to MMP9 levels before chemotherapy, levels of MMP9 after chemotherapy decreased in 61 patients 
TABLE 2: Association of MMP9 serum levels with treatment response.

\begin{tabular}{|c|c|c|c|c|c|c|}
\hline Serum MMP9 & $\begin{array}{c}\mathrm{CR}+\mathrm{PR} \\
\operatorname{Median}(25-75 \%)(\mathrm{ng} / \mathrm{ml})\end{array}$ & $\begin{array}{c}\text { SD+PD } \\
\text { Median }(25-75 \%)(\mathrm{ng} / \mathrm{ml})\end{array}$ & OR (95\% CI) & $P$ & OR (95\% CI) adj & $P$ adj \\
\hline Before treatment & $757.4(546.1-1175.5)$ & $683.5(483.4-1242.3)$ & $1.014(0.938-1.095)$ & 0.728 & $1.032(0.951-1.118)$ & 0.452 \\
\hline After treatment & $449.3(226.4-763.3)$ & $440.5(269.2-640.3)$ & $1.036(0.925-1.160)$ & 0.542 & $1.092(0.983-1.236)$ & 0.164 \\
\hline Change after treatment & $-299.4(-603.1$ to -8.5$)$ & $-286.3(-648.5$ to -74.2$)$ & $1.012(0.925-1.107)$ & 0.797 & $1.020(0.925-1.124)$ & 0.696 \\
\hline
\end{tabular}

Odds ratios (ORs) calculated for an increase of MMP9 levels for $100 \mathrm{ng} / \mathrm{ml}$. Adj: adjusted for C-reactive protein; CR: complete response; PR: partial response; SD: stable disease; PD: progressive disease.

TABLE 3: Association of MMP9 SNPs with serum MMP9 levels before treatment.

\begin{tabular}{|c|c|c|c|}
\hline Genotype & $N(\%)$ & $\begin{array}{c}\text { Serum MMP9 }(\mathrm{ng} / \mathrm{ml}) \\
\text { Median }(27 \%-75 \%)\end{array}$ & $P$ \\
\hline rs17576 c.836A>G, p.Gln279Arg & & & $0.041^{\mathrm{a}}$ \\
\hline AA & 45 (41.7) [2] & $669.8(429.4-973.2)$ & Reference \\
\hline AG & $55(50.9)$ & $698.5(530.2-1244.5)$ & 0.139 \\
\hline GG & $8(7.4)$ & $1313.6(689.4-1591.6)$ & 0.013 \\
\hline $\mathrm{AG}+\mathrm{GG}$ & $63(58.3)$ & $869.6(546.1-1420.2)$ & 0.053 \\
\hline \multicolumn{4}{|l|}{ rs2250889 c.1721C>G, p.Arg574Pro } \\
\hline $\mathrm{CC}$ & 99 (91.7) [2] & $753.8(530.2-1219.1)$ & Reference \\
\hline CG & $9(8.3)$ & $503.0(430.0-1084.7)$ & 0.232 \\
\hline rs17577 c.2003G>A, p.Arg668Gln & & & $0.146^{\mathrm{a}}$ \\
\hline GG & $76(69.7)[1]$ & $661.8(435.9-1130.8)$ & Reference \\
\hline GA & $30(27.5)$ & $1035.5(538.6-1411.7)$ & 0.187 \\
\hline $\mathrm{AA}$ & $2(2.8)$ & $1207.2(820.4-1207.2)$ & 0.120 \\
\hline $\mathrm{GA}+\mathrm{AA}$ & $32(30.3)$ & $1053.5(545.1-1414.5)$ & 0.100 \\
\hline rs 20544 c. $* 3 \mathrm{C}>\mathrm{T}$ & & & $0.222^{\mathrm{a}}$ \\
\hline $\mathrm{CC}$ & $18(16.7)[2]$ & $981.0(558.0-1441.2)$ & Reference \\
\hline $\mathrm{CT}$ & $54(50.0)$ & $721.5(493.9-1225.5)$ & 0.275 \\
\hline $\mathrm{TT}$ & $36(33.3)$ & $687.7(425.2-975.3)$ & 0.093 \\
\hline $\mathrm{CT}+\mathrm{TT}$ & $90(83.3)$ & $702.1(486.8-1176.1)$ & 0.148 \\
\hline
\end{tabular}

${ }^{a}$ Calculated using the Kruskal-Wallis test comparing all three genotypes. Numbers in [] denote missing genotype data.

$(81.3 \%)$ with stable or progressive disease, and in $26(76.5 \%)$ patients with complete and partial response. Increased MMP9 levels were not associated with better response rate $(\mathrm{OR}=1.34,95 \% \mathrm{CI}=0.50-3.58, P=0.559)$, even after adjustment for clinical parameters $(\mathrm{OR}=1.65,95 \% \mathrm{CI}=$ 0.58-4.67, $P=0.345)$.

Genotype frequencies for investigated MMP9 SNPs are presented in Table 3. All were in agreement with HWE $(P>0.050)$.

Among the investigated SNPs, only rs17576 was associated with serum MMP9 levels before treatment $(P=0.041$, Table 3). Carriers of two polymorphic $G$ alleles had significantly higher serum MMP9 level compared to carriers of two wild-type A alleles $(P=0.013)$. The same trend was observed in the dominant model, but the difference failed to reach statistical significance $(P=0.053)$. Furthermore, in haplotype analysis, no significant association of MMP9 haplotypes with serum MMP9 was observed (Supplementary Table 1).

In survival analysis, there were no statistically significant associations of serum MMP9 before treatment, after treatment, and at disease progression with TTP or OS, even after adjustment for clinical parameters (Table 4).

\section{Discussion}

In the present study, we evaluated MMP9 serum expression as an easily accessible serum marker in MM and investigated a possible clinical correlation to OS, TTP, and treatment response. Furthermore, we investigated a correlation between MMP9 serum levels and MMP9 SNPs. We have observed two interesting associations: an association between MMP9 serum levels and ECOG performance status and between MMP9 expression and MMP9 rs17576 polymorphism.

A statistically significant result of our analysis was a correlation between higher MMP9 serum levels before treatment and higher ECOG performance status. Patients that were fully active and able to carry on daily activities without restrictions (ECOG 0) had a significantly lower MMP9 serum concentration in comparison to the patients capable of selfcare, but up and about $50 \%$ of the waking hours (ECOG 2). In a lung cancer study, comparing expression of MMP9 in 


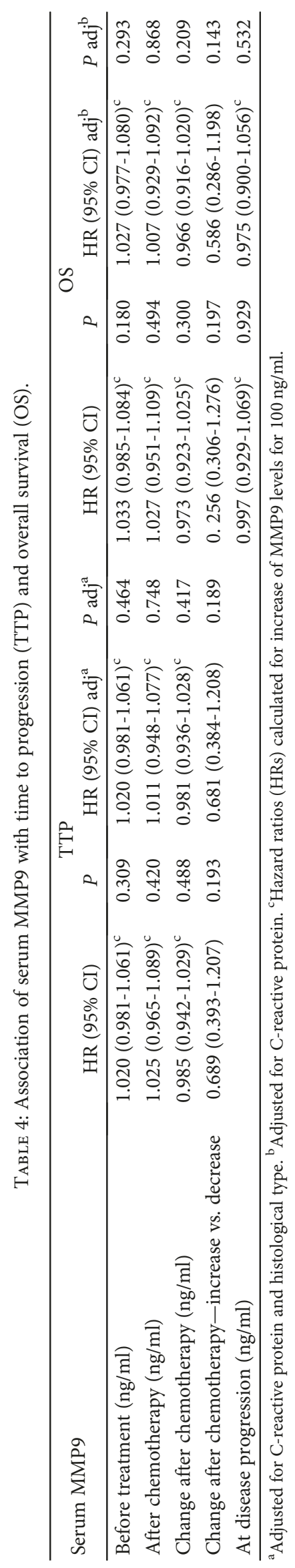


normal vs. cancer tissue MMP9 expression was the highest in adenocarcinoma of greater tumor stage (III and IV). The expression and activity of MMP9 in serum as well as tissue samples correlated with histology and tumor stage, without a reference to performance status [20].

We observed a significant decrease of serum MMP9 after chemotherapy, while the levels rose again at disease progression. Our observations are in agreement with a study in lung cancer concluding that an increase in MMP9 levels after three cycles of chemotherapy was predictive for progression with $5 \%$ increase in the odds of progression for an increase of $10 \mathrm{ng}$ of MMP9 [21]. Also, Qiao et al. showed that differences in serum MMP9 concentration in lung cancer patients before and after disease progression were statistically significant after one, two, and four cycles of chemotherapy [22]. Moreover, a meta-analysis on MMP9 serum concentration in lung cancer, including 26 studies, concluded that MMP9 overexpression correlated with advanced tumor stage and poor 5-year OS. This study noted a difference between the serum and tumor tissue overexpression of MMP9. An overexpression of MMP9 in tumor tissue, but not the serum, had the abovementioned influence on tumor stage and OS [23]. MMP9 has an important role in metastasis of lung adenocarcinoma that was investigated in a recent study. Their conclusion was that an elevated MMP7 and MMP9 play a role in the release of circulating tumor cells into the peripheral blood. The investigators suggested that the concentrations of MMP7 and MMP9 and the circulating tumor cell count could be used together as an effective, clinically predictive panel for lung adenocarcinoma metastasis and prognosis [24]. In other tumors, such as gastric cancer, MMP9 plasma levels correlated better with disease progression and lymph node invasion than the serum levels. Therefore, we believe that further studies are needed to determine the role of serum MMP9 as a diagnostic/prognostic marker [25].

We also set out to investigate a possible link between MMP9 serum concentrations, treatment response to chemotherapy, and an association with TTP and OS in MM patients. We observed some differences in MMP9 serum concentration in patients with complete or partial response and in patients with stable or progressive disease, but these concentration differences failed to reach statistical significance and were not associated with survival or treatment response.

Our study focused on MMP9 concentration in combination with MMP9 polymorphisms as biomarkers in MM. We observed a large interindividual variability in MMP9 serum levels, both before treatment and at different treatment responses; therefore, we investigated the role of genetic variability in MMP9 genes. In our previous study, we established the influence of MMP9 rs2250889 and rs20544 on TTP and OS [18]. In this study, statistically significant association was found between MMP9 rs17576 A $>$ G GG genotype and higher MMP9 serum levels before treatment in comparison to the wild-type MMP9 rs17576 $\mathrm{A}>\mathrm{G}$ AA genotype. This genotype showed a tendency of association with TTP and OS; however, it was not statistically significant.
Our present and our previous studies suggest that MMP9 may play a role in MM. Although MMP9 serum levels failed to reach significant associations with clinical endpoints, therefore we believe that further studies should be performed to elucidate the role of serum MMP9 as a diagnostic/prognostic marker. The limitations of our study was a relatively small sample size, due to the rarity of MM and the lack of comparative data on tumor tissue MMP9 expression levels. However, to the best of our knowledge, this is the first such longitudinal study in MM. Furthermore, we investigated both serum and genetic biomarkers in a well-characterized population of MM patients treated with the same protocols and followed up in the same institution.

Our data suggests that, rather than as standalone biomarkers, MMP9 serum expression as well as MMP9 rs17576 A>G genotype may perform better in combination with other potential biomarkers. Further studies should therefore take into account novel biomarkers, such as microRNAs (miRNAs), high-mobility group box 1 (HMGB1) protein, and proteomic-based assays $[26,27]$. MiRNAs are gaining in interest as potential noninvasive serum biomarkers in several cancers, including MM. For example, miR-126 correlated well with SMRP concentrations in MM patients and the combination was proposed as an early detection biomarker [28]. Proinflammatory cytokine HMGB1 is increased in serum of MM patients, since it drives and sustains MM development and it was proposed as an early detection biomarker and a possible drug target in MM $[29,30]$. Proteomic-based assays simultaneously measuring several proteins could also help to identify novel biomarkers in MM as studies have shown such proteomic signatures could be used in surveillance and diagnosis of MM $[31,32]$. Our future perspective would therefore be to investigate if addition of miRNAs or other protein biomarkers to MMP9 plasma levels and MMP9 rs17576 $A>G$ polymorphism may improve prognostic value of MMP9 in MM.

\section{Conclusions}

In conclusion, we have shown that MMP9 concentration correlates with ECOG performance status and that MMP9 rs17576 influences MMP9 serum concentration in MM patients. However, we believe that MMP9 could have a higher significance as a composite biomarker with other novel biomarkers that are investigated in MM.

\section{Data Availability}

The data used to support the findings of this study are included within the article and supplementary information file.

\section{Conflicts of Interest}

The authors declare no conflict of interest. 


\section{Acknowledgments}

This work was financially supported by the Slovenian Research Agency (ARRS Grant Nos. P1-0170 and L3-8203).

\section{Supplementary Materials}

Supplementary Table 1: association of MMP9 haplotypes with serum MMP9 levels before treatment. (Supplementary Materials)

\section{References}

[1] F. Grosso, N. Steele, S. Novello et al., "Nintedanib plus pemetrexed/cisplatin in patients with malignant pleural mesothelioma: phase II results from the randomized, placebo-controlled lume-meso trial," Journal of Clinical Oncology, vol. 35, no. 31, pp. 3591-3600, 2017.

[2] R. Hassan, A. Thomas, C. Alewine, D. T. Le, E. M. Jaffee, and I. Pastan, "Mesothelin immunotherapy for cancer: ready for prime time?," Journal of Clinical Oncology, vol. 34, no. 34, pp. 4171-4179, 2016.

[3] J. Creaney, I. M. Dick, and B. W. S. Robinson, "Discovery of new biomarkers for malignant mesothelioma," Current Pulmonology Reports, vol. 4, no. 1, pp. 15-21, 2015.

[4] A. Scherpereel, B. Grigoriu, M. Conti et al., "Soluble mesothelin-related peptides in the diagnosis of malignant pleural mesothelioma," American Journal of Respiratory and Critical Care Medicine, vol. 173, no. 10, pp. 1155-1160, 2006.

[5] A. Franko, V. Dolzan, V. Kovac, N. Arneric, and M. DodicFikfak, "Soluble mesothelin-related peptides levels in patients with malignant mesothelioma," Disease Markers, vol. 32, no. 2, 131 pages, 2012.

[6] K. Goričar, V. Kovač, A. Franko, M. Dodič-Fikfak, and V. Dolžan, "Serum survivin levels and outcome of chemotherapy in patients with malignant mesothelioma," Disease Markers, vol. 2015, Article ID 316739, 8 pages, 2015.

[7] V. Kovac, M. Dodic-Fikfak, N. Arneric, V. Dolzan, and A. Franko, "Fibulin-3 as a biomarker of response to treatment in malignant mesothelioma," Radiology and Oncology, vol. 49, no. 3, pp. 279-285, 2015.

[8] A. Cristaudo, A. Bonotti, G. Guglielmi, P. Fallahi, and R. Foddis, "Serum mesothelin and other biomarkers: what have we learned in the last decade?," Journal of Thoracic Disease, vol. 10, no. S2, pp. S353-S359, 2018.

[9] A. J. McCambridge, A. Napolitano, A. S. Mansfield et al., "Progress in the management of malignant pleural mesothelioma in 2017," Journal of Thoracic Oncology, vol. 13, no. 5, pp. 606-623, 2018.

[10] E. Battolla, P. A. Canessa, P. Ferro et al., "Comparison of the diagnostic performance of fibulin-3 and mesothelin in patients with pleural effusions from malignant mesothelioma," Anticancer Res, vol. 37, no. 3, pp. 1387-1392, 2017.

[11] A. Cristaudo, R. Foddis, A. Bonotti et al., "Two novel polymorphisms in $5^{\prime}$ flanking region of the mesothelin gene are associated with soluble mesothelin-related peptide (SMRP) levels," The International Journal of Biological Markers, vol. 26, no. 2, pp. 117-123, 2011.

[12] C. De Santi, P. Pucci, A. Bonotti et al., "Mesothelin promoter variants are associated with increased soluble mesothelinrelated peptide levels in asbestos-exposed individuals,"
Occupational and Environmental Medicine, vol. 74, no. 6, pp. 457-464, 2017.

[13] S. Garritano, C. De Santi, R. Silvestri et al., "A common polymorphism within MSLN affects miR-611 binding site and soluble mesothelin levels in healthy people," Journal of Thoracic Oncology, vol. 9, no. 11, pp. 1662-1668, 2014.

[14] M. Amati, M. Tomasetti, M. Scartozzi et al., "Profiling tumorassociated markers for early detection of malignant mesothelioma: an epidemiologic study," Cancer Epidemiology, Biomarkers \& Prevention, vol. 17, no. 1, pp. 163-170, 2008.

[15] E. L. Servais, C. Colovos, L. Rodriguez et al., "Mesothelin overexpression promotes mesothelioma cell invasion and MMP-9 secretion in an orthotopic mouse model and in epithelioid pleural mesothelioma patients," Clinical Cancer Research, vol. 18, no. 9, pp. 2478-2489, 2012.

[16] A.-C. Buisson, J. M. Zahm, M. Polette et al., "Gelatinase B is involved in the in vitro wound repair of human respiratory epithelium," Journal of Cellular Physiology, vol. 166, no. 2, pp. 413-426, 1996.

[17] D. Strbac, K. Goricar, V. Dolzan, and V. Kovac, "Matrix metalloproteinases polymorphisms as baseline risk predictors in malignant pleural mesothelioma," Radiology and Oncology, vol. 52, no. 2, pp. 160-166, 2018.

[18] D. Štrbac, K. Goričar, V. Dolžan, and V. Kovač, "Matrix metalloproteinases polymorphisms as prognostic biomarkers in malignant pleural mesothelioma," Disease Markers, vol. 2017, Article ID 8069529, 8 pages, 2017.

[19] H.E.Immunoassay, R.E.F.Dep, Quantikine®IVD, ELISA, 2010, July 2018, https://resources.rndsystems.com/pdfs/datasheets/ dtfr1.pdf.

[20] M. K. El-badrawy, A. M. Yousef, D. Shaalan, and A. Z. Elsamanoudy, "Matrix metalloproteinase-9 expression in lung cancer patients and its relation to serum MMP-9 activity, pathologic type, and prognosis," Journal of Bronchology \& Interventional Pulmonology, vol. 21, no. 4, pp. 327-334, 2014.

[21] R. J. Mihaylova Zh1, V. Ludovini, V. Gregorg et al., "Serum level changes of matrix metalloproteinases 2 and 9, vascular endothelial growth factor and epidermal growth factor receptor during platinum-based chemotherapy in advanced non-small cell lung cancer patients," Journal of BUON, vol. 12, pp. 105-111, 2007.

[22] X. Qiao, X. Zhai, J. Wang et al., "Sequential measurements of serum matrix metalloproteinase 9 to monitor chemotherapy responses in patients with advanced non-small-cell lung cancer," OncoTargets and Therapy, vol. 9, pp. 3299-3305, 2016.

[23] L. Gong, D. Wu, J. Zou et al., "Prognostic impact of serum and tissue MMP-9 in non-small cell lung cancer: a systematic review and meta-analysis," Oncotarget, vol. 7, no. 14, pp. 18458-18468, 2016.

[24] Y. Sun, Y. Chen, S. Li et al., "NanoVelcro-captured CTC number concomitant with enhanced serum levels of MMP7 and MMP9 enables accurate prediction of metastasis and poor prognosis in patients with lung adenocarcinoma," International Journal of Nanomedicine, vol. 12, pp. 6399-6412, 2017.

[25] C. Y. Wu, M. S. Wu, E. P. Chiang et al., "Plasma matrix metalloproteinase-9 level is better than serum matrix metalloproteinase-9 level to predict gastric cancer evolution," Clinical Cancer Research, vol. 13, no. 7, pp. 2054-2060, 2007.

[26] R. Foddis, A. Bonotti, S. Landi, P. Fallahi, G. Guglielmi, and A. Cristaudo, "Biomarkers in the prevention and follow-up 
of workers exposed to asbestos," Journal of Thoracic Disease, vol. 10, no. S2, pp. S360-S368, 2018.

[27] H. H. Sun, A. Vaynblat, and H. I. Pass, "Diagnosis and prognosis-review of biomarkers for mesothelioma," Annals of Translational Medicine, vol. 5, no. 11, p. 244, 2017.

[28] L. Santarelli, E. Strafella, S. Staffolani et al., "Association of MiR-126 with soluble mesothelin-related peptides, a marker for malignant mesothelioma," PLoS One, vol. 6, no. 4, pp. e18232-e18239, 2011.

[29] S. Ying, Z. Jiang, X. He et al., "Serum HMGB1 as a potential biomarker for patients with asbestos-related diseases," Disease Markers, vol. 2017, Article ID 5756102, 9 pages, 2017.

[30] L. Pellegrini, J. Xue, D. Larson et al., "HMGB1 targeting by ethyl pyruvate suppresses malignant phenotype of human mesothelioma," Oncotarget, vol. 8, no. 14, pp. 2264922661, 2017.

[31] K. Nagano, "Development and evaluation of antibody proteomics technology for rapid and comprehensive identification of potential biomarkers and therapeutic targets," Biological \& Pharmaceutical Bulletin, vol. 41, no. 5, pp. 663-669, 2018.

[32] R. M. Ostroff, M. R. Mehan, A. Stewart et al., "Early detection of malignant pleural mesothelioma in asbestos-exposed individuals with a noninvasive proteomics-based surveillance tool," PLoS One, vol. 7, no. 10, article e46091, 2012. 


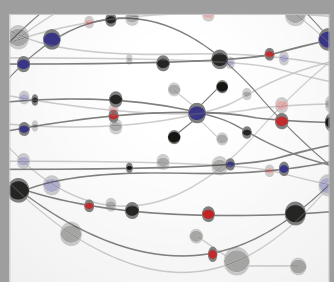

The Scientific World Journal
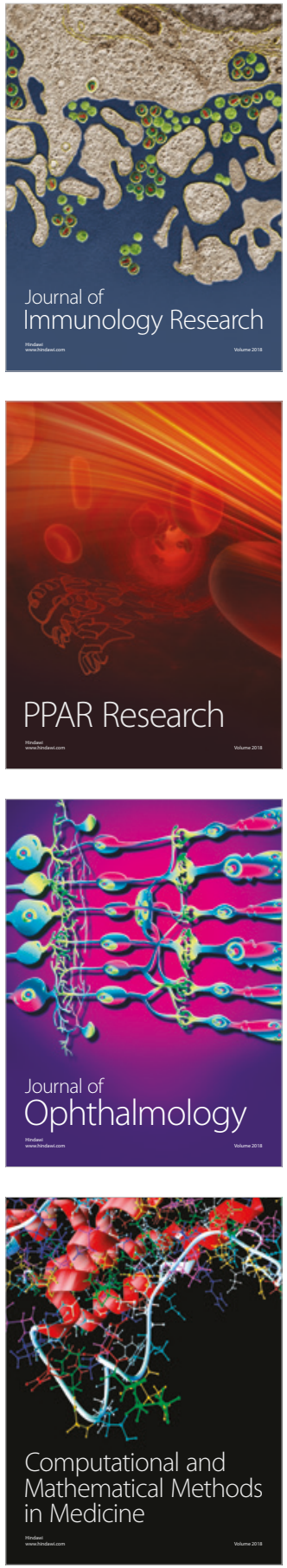

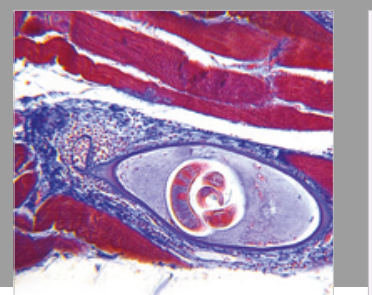

Gastroenterology Research and Practice

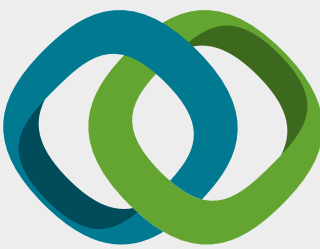

\section{Hindawi}

Submit your manuscripts at

www.hindawi.com


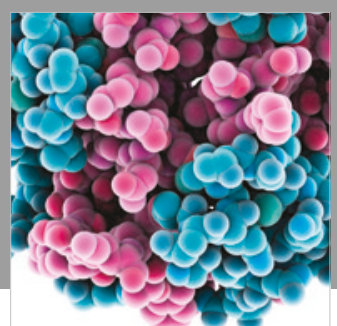

ournal of

Diabetes Research

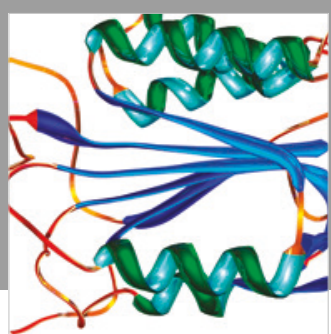

Disease Markers
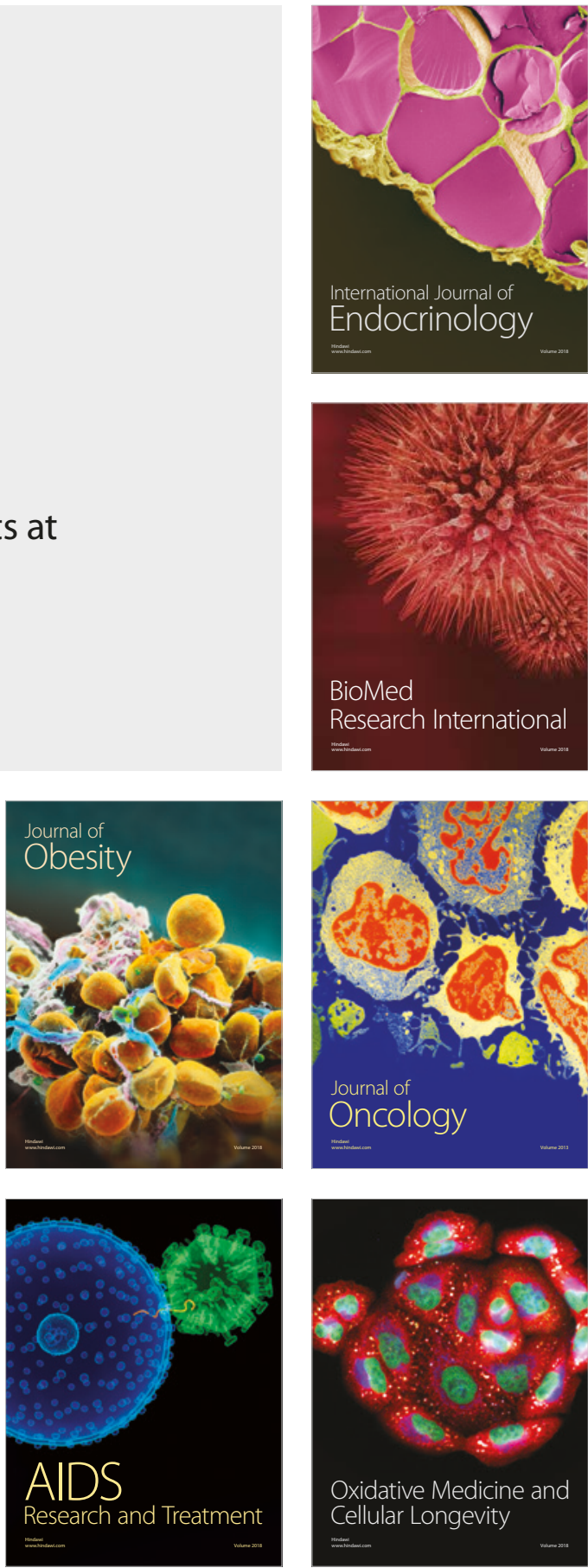